\title{
Publicação contínua e Licença Creative Commons: avanços operacionais na prática editorial da REMAT com vistas à Ciência Aberta
}

\author{
PADILHA, Glauciane Klein Burgiert ${ }^{1,1^{*}}$ \\ ANDREIS, Greice da Silva Lorenzzetti ${ }^{1,2 * *}$ \\ ARCARO, Katia ${ }^{1,3 * * *}$ \\ BOFF, Daiane Scopel ${ }^{1,4 * * * *}$ \\ HAETINGER, Claus ${ }^{1,5 * * * * *}$
}

${ }^{1}$ Instituto Federal de Educação, Ciência e Tecnologia do Rio Grande do Sul, Campus Caxias do Sul - Brasil ORCID ID: https://orcid.org/0000-0001-6843-2289*

ORCID ID: https://orcid.org/0000-0002-8674-0223**

ORCID ID: https://orcid.org/0000-0001-9989-9674***

ORCID ID: https://orcid.org/0000-0002-2860-686X

ORCID ID: https://orcid.org/0000-0003-2633-4052

\begin{abstract}
Resumo
Neste artigo apresenta-se, em uma perspectiva histórica, a trajetória da REMAT: Revista Eletrônica da Matemática ao longo do ano de 2020, considerando sua inserção no campo da editoração e divulgação de pesquisa científica, desde 2015. Com algumas ferramentas conceituais da Ciência Aberta, descrevem-se as principais ações desenvolvidas neste período, tais como a revisão dos direitos autorais dos artigos e a adoção de licenças mais flexíveis, a publicação em fluxo contínuo e o cuidado com a atualização de metadados no ato de cada publicação. Com isso, mapeia-se um caminho que permite inscrever a REMAT em um processo ascendente para a abertura dos ciclos de editoração e publicação de pesquisas junto à comunidade científica.
\end{abstract}

Palavras-chave: Ciência Aberta. Publicação Contínua. Revista de Matemática.

\section{Continuous publication and Creative Commons license: operqtional advances in REMAT's editorial practice aimed at Open Science}

\begin{abstract}
In this paper it has been shown the trajectory of the journal named Revista Eletrônica de Matemática (REMAT) throughout the year 2020, in a historical account, considering its insertion in the field of publishing and dissemination of scientific research, since 2015. Using a few conceptual tools of Open Science, the main actions developed over this period are also described, such as reviewing the copyright of the papers and adopting more flexible licenses, publishing in a continuous flow and taking care to update metadata in the act of each publication. Thereby, a path is mapped that allows to enroll REMAT in an ascending process for the opening of the cycles of publishing and publication of research with the scientific community.
\end{abstract}

Keywords: Open Science. Rolling Pass. Math Journal.

\section{Publicación continua y licencia Creative Commons: avances operativos en la práctica editorial de REMAT orientados a la Ciencia Abierta}

\section{Resumen}

En este trabajo se muestra la trayectoria de la Revista Eletrônica de Matemática (REMAT) a lo largo del año 2020, en un enfoque histórico, considerando su inserción en el campo de la publicación y difusión de la investigación científica, desde 2015. Utilizando algunas herramientas conceptuales de la Ciencia Abierta, también se describen las principales acciones desarrolladas en este período, como revisar los derechos de aut or de los papers y adoptar licencias más flexibles, además de publicar en un flujo continuo y cuidar de la actualización de metadatos en el acto de cada publicación. Siendo así, se mapea un camino que permite inscribir REMAT en un proceso ascendente para la apertura de los ciclos de publicación y publicación de investigaciones con la comunidad científica.

Palabras clave: Ciencia Abierta. Publicación Continua. Revista de Matemáticas. 


\section{Introdução}

A Ciência Aberta está em pauta no meio científico e a discussão acerca do caminho a ser trilhado pelos periódicos vem ganhando força. Segundo Santos e Calò (2020, p. 21),

A Ciência Aberta é frequentemente definida como um conceito abrangente que engloba vários movimentos que buscam a abertura de todo o ciclo de pesquisa e tem o objetivo de remover as barreiras para compartilhar qualquer tipo de resultado, recursos, métodos ou ferramentas empregadas no processo de pesquisa.

Considerando essa perspectiva, são construídas práticas que, ao serem adotadas, contribuem para uma maior rapidez na disponibilização do conteúdo científico, abrindo possibilidades para amplos compartilhamentos e disseminação do conhecimento. A adoção de licenças de direitos autorais mais flexíveis e a publicação em fluxo contínuo, por exemplo, são ações que têm sido incorporadas à rotina de muitos periódicos nacionais e internacionais.

A Creative Commons é um projeto global, sem fins lucrativos, formado por uma rede de voluntários em todo o mundo, que visa oferecer, gratuitamente, licenças de direitos autorais (CREATIVE COMMONS BR, 2020). Segundo Canto, Muriel-Torrado e Pinto (2020, p. 93),

As licenças CC são ferramentas jurídicas que flexibilizam o regramento tradicional de direitos autorais, possibilitando aos autores compartilhar suas obras de acordo com o tipo de uso almejado. As licenças também indicam de forma clara aos usuários o que podem ou não fazer com as obras licenciadas.

No ano de 2020 a REMAT realizou a alteração da licença de CC BY-NC para CC BY. Essa mudança ampliou as possibilidades de uso do que é publicado na Revista. Se antes o conteúdo podia ser usado como referência para criação de novos trabalhos, desde que para fins não comerciais e atribuído o devido crédito de originalidade, agora o conteúdo da REMAT pode ser utilizado, inclusive, para fins comerciais. Essa é a licença mais flexível dentre as disponíveis, sendo "recomendada para maximizar a disseminação e uso dos materiais licenciados" (CANTO; MURIEL-TORRADO; PINTO, 2020, p. 94).

Em relação à forma de disponibilização dos volumes de um periódico, Cirasella e Bowdoin (2013) sugerem que a publicação em "fascículos discretos" pode estar mais alinhada à comercialização do material, no sentido de assegurar a distribuição a seus assinantes de um número fixo de encartes, aproximadamente, do mesmo tamanho. No entanto, os autores defendem que para as revistas de acesso livre e, especialmente, para as disponíveis on-line, a publicação contínua parece ser a mais adequada aos objetivos e preceitos. Ainda em 2013, os autores previram que a publicação contínua tomaria um espaço mais considerável no cenário editorial, tornando-se prevalente nos próximos anos. De fato, essa tem sido uma discussão cada vez mais potente no meio acadêmico e o número de revistas que aderem ao formato rolling pass têm crescido. 
Seguindo essa tendência, a REMAT, em 2020, passou a publicar seu artigos em fluxo contínuo. Até a publicação do volume 5, número 2, em 2019, os artigos eram disponibilizados apenas no fechamento de cada edição, semestralmente. A publicação do volume 6 , número 1 marca essa alteração para o modelo rolling pass. Com a mudança, a periodicidade das edições continua semestral, contudo, os artigos são publicados conforme o processo editorial esteja finalizado. As edições são abertas no primeiro dia dos meses de janeiro e julho e fechadas no último dia dos meses de junho e dezembro. Na sequência, descreve-se o detalhamento de algumas ações realizadas pela REMAT no ano de 2020.

\section{Metodologia}

Este trabalho é de natureza qualitativa e busca fazer um registro histórico acerca das ações realizadas na REMAT no ano de 2020. Historicizar tem, nesse sentido, o objetivo de servir como guia para a compreensão de ações futuras, bem como auxiliar outros periódicos que estão em estágio anterior ou equivalente ao deste. Neste texto, apresentam-se algumas das ações realizadas na REMAT, tais como: a revisão dos direitos autorais dos artigos e a adoção de licenças mais flexíveis, a publicação em fluxo contínuo e o cuidado com a atualização de metadados no ato de cada publicação, e analisa-se seus impactos na pesquisa editorial, tendo por base os preceitos da Ciência Aberta. Todos os dados apresentados e analisados foram extraídos dos registros do sistema de editoração adotado, o Open Journal Systems, versão 3.2.1.0.

\section{Resultados e Discussão}

A REMAT é um periódico eletrônico de acesso livre, que adota o fluxo contínuo de submissões e de publicações, tendo fechamento semestral para suas edições. Adota o Open Journal Systems para sua administração e publicação. Em julho de 2020, a Revista migrou da versão OJS 3.1.1.4 para a versão OJS 3.2.1.0 e, tendo como base os preceitos da Ciência Aberta, estão sendo revisados fluxos e configurações do Sistema.

Nas configurações de distribuição da Revista, no item "Detentor do Copyright" há três possibilidades de escolha: autor, revista e declaração de direitos autorais personalizada. A REMAT, com o intuito de promover a revista, continua retendo os direitos autorais dos artigos publicados, tendo o direito de primeira publicação do trabalho, menção de primeira publicação no periódico nos demais meios publicados e distribuição de partes ou do trabalho como um todo. Essa informação é disponibilizada na página de publicação de cada artigo. Além disso, a REMAT utiliza como data padrão de direitos autorais a data de publicação do artigo. Deter os direitos autorais dos artigos 
permite o compartilhamento e a disseminação das pesquisas dos autores, aumentando o impacto destas e potencializando citações, desejo de seus autores e interesse da Política Editorial da Revista.

Como a REMAT é um periódico em acesso aberto, todo o seu conteúdo está disponível gratuitamente, sem custos para o usuário ou para sua instituição. Com relação às licenças disponíveis no OJS, a REMAT adota a CC Attribution 4.0. Com ela os usuários têm permissão para ler, baixar, copiar, distribuir, imprimir, pesquisar ou vincular os textos completos dos artigos, ou usá-los para qualquer outra finalidade legal, sem solicitar permissão prévia da Revista ou do autor. Esta declaração está de acordo com a definição da BOAI (2002) de acesso aberto. O logo da licença aparece em todas as páginas, bem como na folha de rosto dos artigos publicados pela REMAT. Um entendimento claro sobre os direitos autorais e sobre a licença adotada pelos periódicos é de grande importância no meio editorial, conforme aponta Pimenta et al. (2015, p. 147): "É nítida a necessidade de maior conhecimento e transparência quanto ao uso dos direitos autorais por parte dos periódicos/editoras científicos a fim de se minimizar a insegurança e fragilidade jurídica para a comunidade científica”.

Na área de publicação dos artigos da REMAT são visibilizadas várias informações: nome dos autores, juntamente com sua instituição, registro ORCID e biografia; título; resumo; DOI; referências; arquivo PDF da publicação; data de publicação; número da edição; seção em que o artigo encontra-se publicado; forma de como citar em diferentes formatos, tendo a ABNT como padrão; número de downloads do artigo nos últimos 12 meses; licença adotada pela Revista e a relação de artigos já publicados pelos mesmos autores na REMAT. Sobre isso, Gulka e Silveira (2020, p. 4) apontam que

[...] esses dados acabam por não serem atualizados no próprio sistema de editoração, o que causa inconsistência de informações entre metadados do artigo e sua versão em texto completo, geralmente publicada em formato PDF. Este tipo de prática prejudica a credibilidade e consistência de informações, pois apresentará diferentes conteúdos para representar um único artigo em distintas plataformas de disseminação da revista.

Atentando à questão explicitada, tem-se adotado como prática na REMAT atualizar, no momento da publicação de cada artigo, todas as informações citadas anteriormente, a fim de manter a credibilidade e a consistência dos metadados.

\section{Considerações finais}

Mediante as ações da REMAT apresentadas neste trabalho, percebe-se que muitas são as boas práticas a serem adotadas no viés da Ciência Aberta. Para este ano, a REMAT destaca a revisão dos direitos autorais dos artigos, a adoção de licenças mais flexíveis, a publicação em fluxo contínuo 
e o cuidado com a atualização de metadados no ato de cada publicação. Em especial, entende-se que a publicação em fluxo contínuo responde às expectativas de pesquisadores, autoridades e usuários de informação científica para que novas pesquisas, ensaios e opiniões sejam publicizados o mais rapidamente possível. Ademais, a alteração para o modelo de fluxo contínuo traz agilidade para o sistema de publicações, além de distribuir melhor o trabalho editorial ao longo do ano.

\section{Agradecimentos}

Os autores agradecem o apoio financeiro concedido pela Pró-Reitoria de Pesquisa, Pósgraduação e Inovação (PROPPI) do IFRS por meio do Edital IFRS nº 07/2020 - Apoio à Edição de Periódicos Científicos do IFRS.

\section{Referências}

BOAI. Budapest Open Access Initiative. 14 fev. 2020. Disponível em: https://www.budapestopenaccessinitiative.org/read. Acesso em: 27 ago. 2020.

CANTO, F. L.; MURIEL-TORRADO, E.; PINTO, A. L. Direitos de autor e licenças Creative Commons para periódicos científicos de acesso aberto. In: SILVEIRA, L.; SILVA, F. C. C. (Orgs.). Gestão Editorial de Periódicos Científicos: tendências e boas práticas. 1. ed. Florianópolis: BU Publicações/UFSC, Edições do Bosque/UFSC, 2020, p. 81-104. DOI: https://doi.org/10.5007/978-65-87206-08-0.

CIRASELLA, J.; BOWDOIN, S. Just Roll with It? Rolling Volumes vs. Discrete Issues in Open Access Library and Information Science Journals. Journal of Librarianship and Scholarly Communication, v. 1, n. 4, eP1086, 2013. DOI: https://doi.org/10.7710/2162-3309.1086.

CREATIVE COMMONS BR. Sobre. Disponível em: https://br.creativecommons.org/sobre/. Acesso em: 26 ago. 2020.

GULKA, J. A.; SILVEIRA, L. da. Revisão de metadados para consistência de artigos publicados em acesso aberto. RevIU, v. 2, n. 1, p. 1-10, 2020. Disponível em: http://reviu.febab.org.br/index.php/reviu/article/view/11/10. Acesso em: 28 ago. 2020.

PIMENTA, F. P. et al. O direito de autor no movimento de acesso livre e aberto, um estudo sob a ótica das editoras científicas. Ponto de Acesso, v. 9, n. 3, p. 133-148, dez. 2015. DOI: http://dx.doi.org/10.9771/1981-6766rpa.v9i3.15165.

SANTOS, S. M. dos; CALÒ, L. N. Gestão editorial: tendências e desafios na transição para a ciência aberta. In: SILVEIRA, L.; SILVA, F. C. C. (Orgs.). Gestão Editorial de Periódicos Científicos: tendências e boas práticas. 1. ed. Florianópolis: BU Publicações/UFSC, Edições do Bosque/UFSC, 2020, p. 17-55. DOI: https://doi.org/10.5007/978-65-87206-08-0. 


\section{${ }^{1}$ Glauciane Klein Burgiert Padilha (Caxias do Sul, Rio Grande do Sul, Brasil)}

Licencianda em Matemática pelo Instituto Federal de Educação, Ciência e Tecnologia do Rio Grande do Sul (IFRS), Campus Caxias do Sul, integrante do grupo de pesquisa GPMETA - Grupo de Pesquisa em Matemática, Ensino, Tecnologias e Aplicações, e bolsista de iniciação científica da REMAT: Revista Eletrônica da Matemática. Contribuição de autoria: Escrita - Primeira Redação.

Lattes: http://lattes.cnpq.br/4075849310486386

E-mail: glauciane.padilha@caxias.ifrs.edu.br

${ }^{2}$ Greice da Silva Lorenzzetti Andreis (Caxias do Sul, Rio Grande do Sul, Brasil)

Licenciada em Matemática pela Universidade de Caxias do Sul (2005), mestra em Matemática Aplicada (2008) e doutora em Engenharia Química (2011) pela Universidade Federal do Rio Grande do Sul. Realizou pós-doutorado junto à Universidade Federal do Rio Grande do Sul (2013) na área de combustão, atuando principalmente no desenvolvimento de mecanismos cinéticos reduzidos para biocombustíveis. Atualmente é professora e pesquisadora com dedicação exclusiva do Instituto Federal de Educação, Ciência e Tecnologia do Rio Grande do Sul, Campus Caxias do Sul, integrante dos grupos de pesquisa GPMETA (Grupo de Pesquisa em Matemática, Ensino, Tecnologias e Aplicações) e GPTAI (Grupo de Pesquisa em Tecnologias Aplicadas à Inovação), e editorachefe da REMAT: Revista Eletrônica da Matemática.

Contribuição de autoria: Escrita - Primeira Redação, Revisão e Edição, e Obtenção de Financiamento.

Lattes: http://lattes.cnpq.br/1146104646969214

E-mail: greice.andreis@caxias.ifrs.edu.br

\section{${ }^{3}$ Katia Arcaro (Caxias do Sul, Rio Grande do Sul, Brasil)}

Mestre (2009) e doutora (2013) em Matemática Aplicada, pela Universidade Federal do Rio Grande do Sul, graduou-se em Licenciatura Plena em Matemática com habilitação em Física pela Universidade de Caxias do Sul (2006), com obtenção de Láurea Acadêmica. Atuou como docente da área de Matemática no curso de Administração da Faculdade dos Imigrantes (FAI) e nos cursos de Engenharia de Produção, Engenharia Civil, Engenharia Ambiental, Engenharia Mecânica e Arquitetura e Urbanismo da Faculdade da Serra Gaúcha (FSG). Atualmente, integra o corpo docente da área de Matemática do Instituto Federal do Rio Grande do Sul (IFRS), lotada no Campus Caxias do Sul, e atua como Editora Adjunta na REMAT: Revista Eletrônica da Matemática. Contribuição de autoria: Conceituação e Escrita - Primeira Redação e Revisão.

Lattes: http://lattes.cnpq.br/5613506301677185

E-mail: katia.arcaro@caxias.ifrs.edu.br

\section{${ }^{4}$ Daiane Scopel Boff (Caxias do Sul, Rio Grande do Sul, Brasil)}

Doutora em Educação pela Universidade do Vale do Rio dos Sinos (UNISINOS), na linha de pesquisa Formação de Professores, Currículo e Práticas Pedagógicas. Professora de Matemática do Instituto Federal de Educação, Ciência e Tecnologia do Rio Grande do Sul (IFRS), Campus Caxias do Sul. Editora da REMAT: Revista Eletrônica da Matemática. Integra os grupos de pesquisa: GPMETA (IFRS) - Grupo de Pesquisa em Matemática, Ensino, Tecnologias e Aplicações; e GIPEDI (UNISINOS) - Grupo Interinstitucional de Pesquisa em Docências, Pedagogias e Diferenças.

Contribuição de autoria: Escrita - Primeira Redação e Revisão.

Lattes: http://lattes.cnpq.br/9679635538989977

E-mail: daiane.boff@caxias.ifrs.edu.br

\section{${ }^{5}$ Claus Haetinger (Caxias do Sul, Rio Grande do Sul, Brasil)}

Bacharel em Matemática (UFRGS, 1990), Mestre em Matemática Pura (UFRGS, 1993), Doutor em Matemática Pura (UFRGS, 2000), Especialista em Gestão Universitária (Univates, 2013), Especialista em Gestão de IES (UCS, 2013), Especialista em Gestão Estratégica de Pessoas (Univates, 2015), Licenciado em Formação Pedagógica em Matemática (Uniasselvi, 2020), cursando Especialização em Tecnologias e Ensino de Matemática (Unisselvi). Atualmente é Professor Visitante junto ao IFRS - Campus Caxias do Sul. Atuou como Professor Titular da Universidade do Vale do Taquari - Univates entre 1993 e 2019, nos cursos de graduação das dez Engenharias, bem como nos PPGs em Ambiente e Desenvolvimento, Ensino, e Ensino de Ciências Exatas. Foi Pró-Reitor de Pesquisa, Extensão e Pós-Graduação na UNIVATES (2009-2012). Tem experiência na área de Álgebra NãoComutativa, com ênfase em Teoria de Aneis. Também pesquisa na área de Modelagem Matemática, aplicando-a em questões relacionadas ao meio ambiente. Paralelamente, dedica-se ao Uso de Tecnologias no Ensino de 
Matemática, bem como às Olimpíadas Matemáticas (foi Coordenador Regional da OBM de 2000 a 2019). É editor convidado da REMAT: Revista Eletrônica da Matemática e integra o grupo de pesquisa: GPMETA (IFRS) - Grupo de Pesquisa em Matemática, Ensino, Tecnologias e Aplicações.

Contribuição de autoria: Escrita - Revisão.

Lattes: http://lattes.cnpq.br/6076019811067756

E-mail: claus.haetinger@ caxias.ifrs.edu.br

Avaliador(a) responsável: Cláudia Oliveira de Moura Bueno

\section{Como citar este artigo:}

PADILHA, Glauciane Klein Burgiert, et al. Publicação contínua e Licença Creative Commons: avanços operacionais na prática editorial da REMAT com vistas à Ciência Aberta. In: ABEC Meeting Live, 2020. Anais... São Paulo: Associação Brasileira de Editores Científicos, 2020. DOI: http://dx.doi.org/10.21452/abecmeeting2020.06 